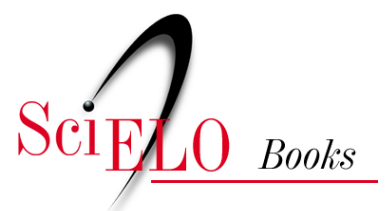

\title{
9. A Abrasco e a Experiência dos Mestrados Profissionais
}

\author{
Eduarda Ângela Pessoa Cesse \\ Maria Amélia de Sousa Mascena Veras
}

\section{SciELO Books / SciELO Livros / SciELO Libros}

CESSE, E.Â.P. and VERAS, M.A.S. A Abrasco e a Experiência dos Mestrados Profissionais. In: LIMA, N.T., SANTANA, J.P., and PAIVA, C.H.A., orgs. Saúde coletiva: a Abrasco em 35 anos de história [online]. Rio de Janeiro: editora FIOCRUZ, 2015, pp. 199-213. ISBN: 978-85-7541-590-0. Available from: doi: $10.7476 / 9788575415900.0011$. Also available in ePUB from: http://books.scielo.org/id/q4gzb/epub/lima-9788575415900.epub.

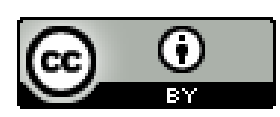

All the contents of this work, except where otherwise noted, is licensed under a Creative Commons Attribution $\underline{4.0 \text { International license. }}$

Todo o conteúdo deste trabalho, exceto quando houver ressalva, é publicado sob a licença Creative Commons Atribição 4.0. 


\title{
A Abrasco e a Experiência dos Mestrados PROFISSIONAIS
}

Eduarda Ângela Pessoa Cesse Maria Amélia de Sousa Mascena Veras

\begin{abstract}
A produção de conhecimentos sobre a educação dos profissionais na área da saúde vem sendo desenvolvida há mais de um século, tendo no Relatório Flexner, de 1910, um marco importante. Este desencadeou muitas mudanças e, em que pesem as críticas e limitações, é considerado ogrande responsável pelas importantes reformas nas escolas médicas nos Estados Unidos da América, com profundas implicações para a formação de profissionais e para a prática da medicina no mundo (Pagliosa \& Da Ros, 2008).

A educação profissional não acompanhou, no mesmo ritmo, os novos desafios apresentados para o setor Saúde, e na primeira década do século XXI ainda persistem inadequações na formação dos profissionais. A fragmentação do currículo, a dificuldade em atualizar conteúdos e a oferta não articulada com as novas necessidades de saúde podem, em parte, explicar a falta de uma resposta adequada a essa lacuna (Frenk et al., 2014).

Tal cenário contribui para manutenção de problemas sistêmicos no setor, muito difíceis de superar de forma equitativa, tais como a incompatibilidade das competências adquiridas ante as necessidades da população; pouco trabalhoem equipe; ênfaseno componentetécnico; encontros episódicos, em vez de cuidados continuados; orientação hospitalar predominante em detrimento da atenção primária; desequilíbrios quantitativos e qualitativos no mercado de trabalho profissional; e fraca liderança para melhorar o desempenho do sistema de saúde (Frenk et al., 2014).

Diante de antigas necessidades e novos desafios, os sistemas de saúdeem todo o mundo estão se tornando mais complexos e caros, demandando maior número de profissionais qualificados. Em se tratando de gestores dos serviços de saúde, tal insuficiência torna-se ainda
\end{abstract}


mais evidente, na medida em que a cada dia é necessário incorporar novas ferramentas para o aperfeiçoamento dos serviços ofertados à população.

Atualmente, no Brasil, o processo de formação de recursos humanos dealta qualificação na área da saúde compreende diversos níveis de formação, ofertados por instituições nas diferentes regiões.São cursos deespecializaçãolato sensu nos diferentes campos disciplinares, emestrados, profissionais ou acadêmicos, edoutorados vêm consolidando o sistema brasileiro de pós-graduação stricto sensu (Goldbaum, 2006). No entanto, a complexidade sanitária e a consolidação de um sistema com as características do Sistema Único de Saúde (SUS) requerem a urgência na formação de profissionais com perfis de especialização distintos dos tradicionais e com características diferentes das disponíveis no sistema de pós-graduação do país (Hartz \& Nunes, 2006). Tais diferenças devem ser expressas na orientação dos currículos, na composição dos corpos docente e discente, bem como nas formas de financiamento e novos arranjos institucionais (Mestrado no Brasil, 1995).

Nessa perspectiva, o mestrado profissional (MP) emerge na década de 1990 como uma opção de formação pós-graduada no país que se destina à capacitação operacional e ao desenvolvimento e inovação aplicáveis, de modo direto e efetivo, ao campo de atuação do profissional em formação (Quelhas, Faria Filho \& França, 2005). Vale salientar que a emergência dos MPs no Brasil corrobora a larga história de educação profissional que é hoje reconhecida e tratada como uma política de Estado, tendo em vista todo o processo de desenvolvimento que no país vem ocorrendo, acompanhando a tendência mundial (Ribeiro, 2006).

\section{A Abrasco e o Surgimento dos Mestrados Profissionais no Brasil: RESISTÊNCIAS E NECESSIDADES DE ADEQUAÇÃO À SAÚDE}

Até a década de 1970, a formação em saúde pública no Brasil se dava por meio dos cursos de especialização. As características dos cursos de mestrado e doutorado só foram definidas e fixadas pelo parecer n. 977/65 (Brasil, 1965), do Conselho Federal de Educação, conhecido como Parecer Sucupira, e basearam-se no modelo norte-americano de formação pós-graduada.

Além de responderà necessidade de implantare desenvolver cursos de pós-graduação, esse parecer traz a distinção entre a pós-graduação stricto sensu e lato sensu. Nesta, os cursos de especialização e aperfeiçoamento têm objetivo técnico profissional específico, sem abranger o campo total do saber em que se insere a especialidade. São destinados ao treinamento nos conteúdos específicos que compõem um ramo profissional ou científico. Já a pós-graduação stricto sensu confere grau acadêmico, que deverá ser atestado por meio de uma alta competência científica em determinada área do conhecimento.

Vale salientar que a pós-graduação brasileira foi formulada nesse parecer como um sistema com dois eixos de formação, o acadêmico e o profissional, porém apenas o eixo 
acadêmico foi implementado integralmente. Só mais recentemente a oferta de MPs vem colocando em prática o eixo profissional, embora tenha sofrido grande rejeição de algumas áreas. Como fatores desafiadores, observam-se imprecisões acerca dos conceitos de cursos acadêmicos e profissionais, dilemas em torno dos componentes estruturais e das diferenças entre as duas modalidades de formação pós-graduada (Fischer, 2010).

No contexto da definição e fixação das características da pós-graduação stricto sensu no país, a Associação Brasileira de Pós-Graduação em Saúde Coletiva (Abrasco) é criada no final da década de 1970. A entidade hoje é intitulada Associação Brasileira de Saúde Coletiva, incluindo a recém-criada graduação em saúde coletiva no país. A Abrasco não só foi estratégica para o processo de redemocratização, a Reforma Sanitária brasileira e a formulação doSUS, como também vem desempenhando um papel importante na formação de recursos humanos (Fonseca, 2006). Notadamente, a criação da graduação em saúde coletiva colocou em debate um conjunto de questões envolvendo a constituição e os desdobramentos da profissão de sanitarista (Paim \& Pinto, 2013), aumentando a responsabilidade da associação nesse novo cenário de formação profissional.

Nota-se que a capacidade de atuação acadêmica da Abrasco em fortalecer os vínculos entre esse campo de formação profissional e as mudanças políticas e institucionais em curso naárea da saúde pública pressupunha a articulação entre as diferentes categorias profissionais. Além disso, vislumbrava-se a construção de competências específicas nas diferentes carreiras da saúde, como também o conhecimento do modelo gerencial proposto e aprovado para o sistema de saúde que, naquele momento, se formatava. Destacava-se a necessidade de conciliar as exigências do mundo acadêmico e da excessiva especialização com as demandas identificadas nos serviços públicos de saúde (Fonseca, 2006).

No momento de criação da Abrasco, a pesquisa científica no campo também era pouco expressiva e subfinanciada. O desenvolvimento da pós-graduação no país ainda era relativamente incipiente, com cursos de mestrado e doutorado concentrados em apenas três estados: São Paulo, Rio de Janeiro e Bahia. A formação profissional era dada por meio de cursos de especialização em saúde pública oferecidos pela Faculdade de Saúde Pública da Universidade de São Paulo (USP) e pela Escola Nacional de Saúde Pública da Fundação Oswaldo Cruz (Ensp/Fiocruz). As residências médicas em medicina preventiva emedicina social eram oferecidas por alguns departamentos de escolas médicas, também geograficamente concentrados na região Sudeste (Goldbaum \& Barata, 2006; Fonseca, 2006).

Vale destacar que o processo de avaliação implantado pela Coordenação de Aperfeiçoamento de Pessoal de Nível Superior (Capes) no final da década de 1970 não tinha definição clara de indicadores. Consequentemente a avaliação era muito subjetiva, embora, na atualidade, o debate recaia sobre o caráter excessivamente quantitativo dos critérios utilizados por esse órgão. 
Em 1994, a Abrasco criou o Fórum de Coordenadores dos Programas de Pós-Graduação em Saúde Coletiva (Goldbaum \& Barata, 2006), que, desde então, vemse constituindo em um espaço estratégico para aprofundar as questões relacionadas à pós-graduação. Tem entre seus objetivos buscar maior homogeneidade entre os programas e, fundamentalmente, contribuir para fortalecer o seu desenvolvimento, articulando o trabalho da associação ao da representação de área na Capes, estabelecendo assim um canal permanente de comunicação entre eles. Além do Fórum da Abrasco, no âmbito dos MPs, destaca-se o papel de espaços como o Fórum Nacional de Mestrados Profissionais (Foprof), que permitem uma discussão ampla envolvendo as características dos MPs oferecidos em diferentes áreas de conhecimento, enriquecendo o debate e a busca de resoluções em torno de questões comuns nessa modalidade de formação.

Portanto, a Abrasco vem mantendo o desafio de, no âmbito da formação profissional, contribuir para a discussão da contínua preparação de recursos humanos com capacidade técnica para identificar e hierarquizar as necessidades sociais em saúde; organizar saberes e instrumentos em modelos tecnológicos de trabalho que garantam a efetividade das intervenções; e avaliar os impactos das intervenções adotadas. Além da capacidade técnica, os profissionais da saúde coletiva necessitam ser capazes de: realizar a negociação política interna e externa ao setor; fazer a intermediação entre o conhecimento acadêmico e as práticas nos serviços de saúde; e organizar as evidências científicas para embasar as funções regulatórias do Estado (Fonseca, 2006).

Tendo em vista a sua missão de promover o pensamento e a prática de saúde pública, a Abrasco, no final da década de 1990, promoveu um processo de investigação com o objetivo de abranger questões que a avaliação da Capes, por ter objetivos diferentes, não conseguia aprofundar. Dessa forma, foi possível identificar a falta de clareza e de adequação dos cursos ao perfil da demanda, principalmente nos cursos de mestrado acadêmico, cuja clientela era, na maioria, formada por profissionais inseridos nos serviços do SUS ou seus gestores (Minayo, 2006). Essa observação desencadeou um importante debate sobre o MP como uma possibilidade de formação de profissionais mais interessados em adquirir instrumentos conceituais e técnicos voltados para a prática do que em seguir carreira de pesquisadores (Minayo, 2006).

Assim, o debate em torno dessa modalidade de formação estava aberto, mas a regulamentação dos MPs no Brasil só tem início com a portaria n. 47, de outubro de 1995 (Capes, 2005b), no mesmo momento em que a Capes lança o documento "Programa de Flexibilização do Modelo de Pós-graduação Senso Estrito em Nível de Mestrado" (Capes, 2005c). Em 1998, a portaria n. 80 (Capes, 1998), que dispõe sobre os MPs, apresenta como um de seus principais objetivos"promover o conhecimento científico por meio da prática no meio profissional", e em 2009 a portaria normativa n. 17 (Capes, 2009) define essa modalidade como formação de pós-graduação stricto sensu. Esta confere título com validade nacional, 
com idênticos grau e prerrogativas, inclusive para o exercício da docência, do título de mestre concedido pelos demais programas de pós-graduação stricto sensu. Desdeentão, os MPs vêm ganhando destaque no âmbito da formação de recursos humanos qualificados para enfrentar osnovosdesafiosapresentados pelos processos detrabalhonasociedadeem desenvolvimento. O reconhecimento da propriedade do MP como indicador de inserção social e incremento da demanda, tanto no setor privado quanto no setor público, que buscam qualificar seus quadros, bem como a indução do Ministério da Educação, explicam o crescimento da oferta dessa modalidade de ensino em tão curto espaço de tempo.

Entre 1995, quando a portaria n. 47 foi publicada, e os dias atuais, o debate tem avançado, e em algumas áreas já se vislumbra uma substituição gradativa dos mestrados acadêmicos pela modalidade profissional. Apesar de esse movimento não ser consensual, não restam dúvidas sobre a importância de se aumentar a oferta de MPs em todas as áreas. No campo da saúde coletiva, tais cursos vêm garantindo o seu espaço acompanhando a necessidade de fortalecimento dos quadros para o SUS.

No contexto apresentado, o MP surge para responder à carência de profissionais pós-graduados aptos a elaborarem novas técnicas e processos de trabalho, mais focados em respostas às questões e aos problemas apresentados pelo trabalho. A formação profissional difere da formação acadêmica, que visa, preferencialmente, a aprofundar conhecimentos ou técnicas no campo da produção de conhecimento.

Essa modalidade de formação pós-graduada não poderia encontrar campo mais adequado para ser absorvida do que a saúde coletiva do Brasil, no século XX. Diante do desafio de avançarmos da concepção para a implementação de um sistema de saúde com as características e dimensões do SUS, são necessários profissionais qualificados para responder adequadamente, de forma criativa e cientificamente alicerçada, aos desafios apresentados.

A despeito do fato de a área da saúde coletiva constituir um campo de produção de saberes e práticas, eminentemente aplicados à solução de problemas, nem sempre a ideia do MP foi vista como atraente pelos cursos e programas acadêmicos. No final da década de 1990, havia ainda muita resistência por parte dos coordenadores de programas e das autoridades acadêmicas nas universidades públicas em adotar essa modalidade de formação. Poucas foram as unidades acadêmicas da área da saúde coletiva que apoiaram a proposta em seu início. A primeira proposta na área foi aprovada pela Capes em 2000. Naquele momento, já havia 27 programas em funcionamento em outras áreas do conhecimento, dos quais cinco em odontologia, pioneira na oferta de MP na grande área da saúde.

As primeiras instituições que decidiram oferecer MPs já tinham programas de pósgraduação consolidados e de ótima qualidade: o Instituto de Medicina Social da Universidade do Estado do Rio de Janeiro (2000), o Instituto de Saúde Coletiva da Universidade Federal da Bahia (2001) e a Ensp/Fiocruz (2002). Assim, os primeiros MPs na área nasceram sob a influência de programas acadêmicos muito bons, o que lhes conferiu qualidade e solidez. 
Na atualidade, os MPs têm o reconhecimento da comunidade científica. O Conselho Técnico Científico da Educação Superior (CTC-ES), em sua 148 reunião, realizada em agosto de 2013, criou o cargo de coordenador adjunto de área para MP, além de uma agenda para o desenvolvimento de programas profissionais em todas as áreas de conhecimento.

O maior número de MPs é nas áreas interdisciplinar, ensino, administração e saúde coletiva. Em termos proporcionais, a área da saúde coletiva é a segunda em MPs, sendo $41,6 \%$ dos seus programas oferecidos nessa modalidade, ficando atrás apenas da área de ensino, na qual 57,3\% dos programas são mestrados profissionais (Capes, 2014a, 2014b). Apesar de seu reconhecimento econsolidação na saúde coletiva, percebem-seinconsistências em algumas propostas e falta de definições claras acerca de características que fazem esses cursos realmente voltados para uma formação profissional sólida e para o enfrentamento dos desafios apresentados ao setor Saúde.

Por meio da análise das fichas de avaliação dos MPs em funcionamento na última avaliação trienal da Capes realizada em 2013, foi possível verificar que persistem alguns problemas estruturais a serem enfrentados pelo órgão avaliador da pós-graduação e pelas instituições que ofertam os cursos profissionais, que são as instituições de ensino superior (IES), os centros de pesquisas públicos e privados.

Foi possível identificar que os objetivos não estão claramente especificados e voltados para o campo profissional em todos esses mestrados. Também se verificou que nem sempre os objetivos são coerentes com a estrutura do curso, suas áreas de concentração, linhas de pesquisa e organização disciplinar.

No entanto, o momento de afirmação e consolidação dos MPs requer a identificação de elementos claros das competências de formação profissional como forma de melhor caracterizar a identidade dessa modalidade de formação, diferenciando-a da formação acadêmica já consolidada. Essa necessidade é ainda mais urgente no momento em que a Capes anuncia que fará ajustes no sistema de avaliação, já iniciados com o lançamento da Plataforma Sucupira, bem como realizará adequações em alguns mestrados hoje acadêmicos que passarão a ser profissionais e vice-versa, além de criar o doutorado profissional, previsto no Plano Nacional de Pós-Graduação, mas ainda sem regulamentação estabelecida.

Houve mudanças importantes no que se refere ao financiamento, que na área da saúde coletiva era considerado um item importante para a sua aprovação. Ao longo do tempo, o financiamento de um órgão demandante do MP (secretarias municipais, estaduais de Saúde etc.) paulatinamente deixou de ser requisito para a abertura de MPs - o que pode ser evidenciado na análise das fichas de avalição dos cursos atualmente ofertados. Foi possível observar que nem sempre estavam presentes na proposta do MP os mecanismos de articulação com secretarias estaduais e municipais de Saúde e com o Ministério da Saúde embora seja imprescindível a articulação entre as IES e os centros de pesquisas e os serviços de saúde (municipais, estaduais e Ministério da Saúde), como forma de dar sustentabilidade 
ao curso. Demandantes e ofertantes deverão construir as propostas em conjunto como forma de alcançar o objetivo de preparar profissionais para o enfrentamento dos desafios do sistema de saúde.

No Quadro 1, sintetizam-se os principais problemas que têm dificultado o bom andamento dos MPs e identificam-se suas consequências.

\section{Quadro 1 - Problemas e consequências identificadas nos atuais mestrados profissionais na área da saúde coletiva}

\begin{tabular}{|c|c|}
\hline Problemas & Consequências \\
\hline \multicolumn{2}{|l|}{ 1. Proposta dO PROGRAMA } \\
\hline $\begin{array}{l}\text { Distribuição desigual ou sobreposição das linhas de } \\
\text { pesquisa entre as áreas de concentração. }\end{array}$ & \multirow{4}{*}{$\begin{array}{l}\text { Proposta de programa inconsistente e instável; } \\
\text { incoerência entre a finalidade da oferta, os objetivos e } \\
\text { o perfil do egresso; problemas com a sustentabilidade } \\
\text { financeira do programa e com o desenvolvimento de } \\
\text { pesquisa aplicada aos serviços; dificuldade em atender } \\
\text { a demandas sociais e profissionais da região. }\end{array}$} \\
\hline Projetos desarticulados das linhas de pesquisa. & \\
\hline $\begin{array}{l}\text { Mudanças na estruturação das áreas de } \\
\text { concentração e das linhas de pesquisa entre etapas } \\
\text { avaliativas do programa. }\end{array}$ & \\
\hline $\begin{array}{l}\text { Ausência de mecanismos de articulação com } \\
\text { secretarias estaduais e municipais de Saúde e } \\
\text { com o Ministério da Saúde. }\end{array}$ & \\
\hline \multicolumn{2}{|l|}{ 2. ESTRUTURA CURRICULAR } \\
\hline $\begin{array}{l}\text { Desequilíbrio entre disciplinas teóricas, } \\
\text { metodológicas e instrumentais. }\end{array}$ & \multirow{2}{*}{$\begin{array}{l}\text { Pouca diferenciação entre oferta de um curso lato } \\
\text { sensu e stricto sensu; não apropriação pelos alunos de } \\
\text { conteúdos de metodologia científica indispensável } \\
\text { para a incorporação da ciência na prática profissional; } \\
\text { matriz curricular não ajustada aos objetivos, áreas de } \\
\text { concentração e linhas de pesquisa do curso. }\end{array}$} \\
\hline $\begin{array}{l}\text { Matriz curricular não ajustada aos objetivos, às } \\
\text { áreas de concentração e às linhas de pesquisa. }\end{array}$ & \\
\hline \multicolumn{2}{|l|}{ 3. CORPO DISCENTE } \\
\hline Aluno não inserido nos serviços de saúde. & $\begin{array}{l}\text { Ausência de diálogo com o SUS e, } \\
\text { consequentemente, qualificação inadequada e } \\
\text { insuficiente de profissionais para instituições } \\
\text { públicas da área da saúde. }\end{array}$ \\
\hline \multicolumn{2}{|l|}{ 4. CORPO DOCENTE } \\
\hline Participação em quatro ou mais programas. & \multirow{5}{*}{$\begin{array}{l}\text { Corpo docente em situação irregular de acordo com o } \\
\text { estabelecido na portaria n. 1/2012 (Capes, 2012); corpo } \\
\text { docente instável e frágil para dar suporte aos objetivos } \\
\text { do curso, comprometendo a formação de profissionais } \\
\text { na área; corpo docente permanente responsável por } \\
\text { pequena proporção de atividades nucleares do curso, } \\
\text { sugerindo dependência dos docentes colaboradores. }\end{array}$} \\
\hline $\begin{array}{l}\text { Desequilíbrio na participação em atividades } \\
\text { nucleares do programa (docência, pesquisa e } \\
\text { orientação). }\end{array}$ & \\
\hline Falta de qualificação no campo da saúde coletiva. & \\
\hline $\begin{array}{l}\text { Desequilíbrio entre excelência acadêmica e } \\
\text { experiência profissional. }\end{array}$ & \\
\hline Corpo docente instável. & \\
\hline \multicolumn{2}{|l|}{ 5. ProduÇão BIBLIOGRÁFICA E TÉCNICA } \\
\hline $\begin{array}{l}\text { Produção bibliográfica e técnica desarticulada e } \\
\text { incoerente com os objetivos e linhas de atuação } \\
\text { do programa. }\end{array}$ & $\begin{array}{l}\text { Impacto social, tecnológico e sanitário negativo na } \\
\text { formação de profissionais pouco integrados ao SUS. }\end{array}$ \\
\hline
\end{tabular}


Quadro 1 - Problemas e consequências identificadas nos atuais mestrados profissionais na área da saúde coletiva (cont.)

\begin{tabular}{|c|c|}
\hline Problemas & Consequências \\
\hline $\begin{array}{l}\text { 6. TRABALHOS DE CONCLUSÃo (AVALIADOS PELOS } \\
\text { RESUMOS) }\end{array}$ & \\
\hline $\begin{array}{l}\text { Trabalhos de conclusão com características } \\
\text { acadêmicas. }\end{array}$ & \multirow{2}{*}{$\begin{array}{l}\text { Trabalhos de conclusão sem potencial de aplicação no } \\
\text { sistema de saúde e incoerentes em relação aos objetivos } \\
\text { do programa. }\end{array}$} \\
\hline $\begin{array}{l}\text { Temáticas dos trabalhos de conclusão do curso sem } \\
\text { vínculo com as linhas de pesquisa do programa. }\end{array}$ & \\
\hline
\end{tabular}

Fonte: Elaboração própria com base nas fichas de avaliação trienal 2013, Capes. Disponíveis em: <http://conteudoweb.capes.gov. $\mathrm{br} /$ conteudoweb/CadernoAvaliacaoServlet?acao=filtraArquivo\&ano=2012\&codigo_ies=\&area=22>. Acesso em: set. 2014.

\section{Desafios para a Consolidação do Mestrado Profissional na Saúde Coletiva}

$\mathrm{O}$ MP, tal como organizado na atualidade, e diante do crescimento em todas as áreas de conhecimento, embora ainda guarde resistência em algumas, no campo da saúde coletiva é responsável pela constituição de uma inteligência técnica e política para atuação no SUS.

A formatação e a implantação dos cursos de MP na área da saúde coletiva têm suscitado um incessante debate, que não representou qualquer obstáculo à sua franca expansão; ao contrário, vem servindo para aperfeiçoar de modo contínuo essa modalidade de formação. A Abrasco desempenha papel relevante, ao assegurar espaço nos seus principais eventos para que o debate sobre o MP continue fundamentalmente no âmbito do Fórum de PósGraduação em Saúde Coletiva - que em reuniões plenárias com o conjunto de coordenadores de programas de pós-graduação da área tem contribuído para refinar os instrumentos de avaliação, de tal forma que as especificidades da área estejam contempladas.

Apesar dosavançosalcançados pormeio da regulamentação,énecessário consolidaros cursos de MP para que se tornem a opção de formação para profissionais aptos a enfrentarem estrategicamente os desafios do campo da saúde coletiva. Algumas características são desafiadoras do ponto de vista operacional, bem como em razão de práticas já estabelecidas que requerem mudança na forma de execução dos programas existentes e daqueles a serem propostos.

Um dos objetivos dessa modalidade de formação pós-graduada é, além dos já mencionados no capítulo, expandir a pós-graduação para ambientes não acadêmicos. Este objetivo tem sido mais difícil de atingir, não apenas na área da saúde coletiva. Até o início de 2015, apenas três cursos eram oferecidos por serviços de saúde ou instituições diretamente ligadas aos gestores do SUS municipal, estadual ou federal. Todos os demais eram oferecidos por IES ou institutos de pesquisa. 
Chamaaatençãoo fatodeessamodalidade, apesardeduas décadas deestabelecimento, ainda não ter alcançado um consenso quanto à sua natureza, seus produtos e seu impacto na sociedade brasileira (Hortale, 2006; Santos, Hortale \& Arouca, 2012). É o contrário do que ocorre, por exemplo, em algumas das principais universidades norte-americanas, nas quais o mestrado profissional, chamado mestrado em saúde pública (master in public health - MPH), encontra-se bem consolidado - definido de maneira geral como um grau profissional baseado na prática, que prepara os alunos para serem líderes em um ambiente de saúde pública, qualificados para as posições nos departamentos de saúde nos níveis federal, estadual e locais; nas organizações de base comunitária; em organizações de cuidados de saúde; e em institutos de pesquisa, entre outras instituições. Os cursos de MPH têm em geral duração de dois anos, mas parte dessas universidades oferece um programa em um ano para algumas áreas de estudo, destinado a estudantes que já apresentam grau equivalente ao doutoramento, como os médicos, ou que estejam matriculados simultaneamente em um programa de doutorado.

Os desafios atuais para avançar na consolidação da formação profissional sob forma de MP incluem a identificação de suas características pedagógicas; o aperfeiçoamento dos mecanismos de avaliação e das fontes de financiamento; a superação de assimetrias regionais; e a oferta de MPs em rede.

Do ponto de vista pedagógico e em consonância com os seus objetivos, a proposta do MP deve se diferenciar do mestrado acadêmico, em que tem prevalecido a estrutura disciplinar, privilegiando a constituição de áreas temáticas, com especificação mais clara dos problemas e práticas envolvidos em recortes específicos - como é o caso da gestão de sistemas de saúde, epidemiologia em serviços de saúde e vigilância sanitária, por exemplo (Teixeira, 2006). As características do seu corpo discente, formado por profissionais que estejam inseridos em atividades dos serviços, exigem flexibilidade na constituição das suas atividades acadêmicas, sendo recomendável pensar em atividades de ensino a distância, em combinação com as presenciais.

Os trabalhos de conclusão dos MPs devem ser distintos dos tradicionais, para que haja coerência com a sua proposta e as abordagens pedagógicas. Dessa forma, os produtos finais podem ser:estudos de sínteses que subsidiem a formulação de políticas e projetos; textos que contenham os resultados da elaboração e experimentação de instrumentos de trabalho e de tecnologias leves, a serem incorporados à gestão, promoção ou prevenção de riscos eagravos; ou estudos de caso no âmbito das organizações de saúde, cujos resultados contribuam para a introdução de inovações gerenciais, organizativas e operacionais no processo de produção de ações e serviços.

Nesse sentido, apesar de a portaria n. 17, de 28 de dezembro de 2009 (Capes, 2009), normatizar diversas possibilidades de produto final para o MP, ainda é um desafio para os programas e alunos desenvolverem seus trabalhos de forma diferente da tradicional. 
Mecanismos mais efetivos de indução, bem como espaços de divulgação que privilegiem formatos diversificados, também precisam ser incentivados. Quanto ao corpo docente, a formação na modalidade profissional exige que ele seja altamente qualificado, o que pode ser demonstrado pela produção intelectual constituída por publicações específicas, produção artística ou produção técnico-científica, ou ainda por reconhecida experiência profissional, mesmo na ausência de titulação acadêmica, quando for o caso.

Hartz e Nunes (2006) destacam que, por se tratar de um mestrado com perspectiva multidisciplinar, consubstanciada na articulação de diferentes campos de conhecimento, deve-se contar com a participação de professores com formaçãoe experiência de trabalho nos respectivos campos relacionados. Odesafio diante dessa questão está na excessiva valorização da produção científica tradicional. Nesse contexto, há a necessidade de se aprofundarem normas específicas e critérios adequados de avaliação. Para programas cuja vocação é uma produção de conhecimento de natureza aplicada, é vital a formação de parcerias com o setor extra-acadêmico, de modo a se assegurar não só a criação de novas tecnologias, mas também a sua aplicação.

Com base nas avaliações anteriores, o Fórum de Coordenadores de Pós-Graduação em Saúde Coletiva propôs agrupar a produção técnica oriunda dos programas de pós-graduação em quatro eixos: produção de material bibliográfico com foco técnico-instrucional; produtos técnicos de natureza instrumental; disseminação do conhecimento; e serviços técnicos especializados. A definição desses eixos levou em consideração critérios como abrangência, complexidade (esforço intelectual), aplicabilidade social e econômica e impacto. Esta é uma contribuição que visa a aperfeiçoar o processo de avaliação e que se encontra em debate no momento.

Diante do crescimento da oferta de programas profissionais, observa-se a necessidade de aperfeiçoar o processo de avaliação para que continue servindo de instrumento de busca e manutenção de um padrão de excelência da pós-graduação no país. Em 2014, a Plataforma Sucupira entrou em funcionamento e promete maior agilidade no processo avaliativo, com a alimentação dos dados pelos programas de forma contínua. Torna-se oportuno que essa plataforma permita a geração de relatórios e bancos de dados que possibilitem aos programas realizarem análises autoavaliativas, extremamente úteis, principalmente para os programas menos consolidados.

Conforme recomenda o Plano Nacional de Pós-Graduação (PNPG) 2011-2020 (Capes, 2010), os princípios que nortearão o sistema de avaliação da próxima década são a diversidade e a busca pelo contínuo aperfeiçoamento, que deverão ser observados pelos comitês e pelas instâncias superiores.

O crescimento da área entre 1970 e 2012 é indiscutível. Se esse crescimento for mantido, serão necessários processos avaliativos que valorizem aspectos qualitativos, embora a utilização de indicadores quantitativos deva permancer. Projeta-se para 2020 a existência 
de 142 cursos na área. Assim, a avaliação precisará estar baseada na qualidade e excelência dos resultados, na especificidade das áreas de conhecimento e no impacto dos resultados nas comunidades acadêmica e empresarial e na sociedade.

O PNPG 2011-2020 ressalta que um dos principais desafios está na periodicidade das avaliações (Capes, 2010). A ampliação do tempo de avaliação para os programas mais consolidados e redução do tempo para aqueles mais incipientes e com maiores dificuldades estão entre as metas a serem perseguidas. Assim, as recomendações do PNPG atuam em duas frentes: aumentar a eficiência dos processos e induzir ações específicas para melhorar outras áreas. Como exemplos das melhorias que podem ser estimuladas, o PNPG identifica oportunidades nas dimensões da educação básica, da formação de recursos humanos para setores governamentais (particularmente importante na área da saúde coletiva no que se refere aos MPs) e não governamentais, bem como na indução de áreas de conhecimento específicas.

Quanto aos mecanismos definanciamento, os primeiros cursos de MPforam propostos e avaliados considerando-se a existência de um demandante, instituição/organização responsável por financiar as atividades. Embora tal característica não seja na atualidade uma exigência, torna-se oportuna uma estreita articulação entre o ofertante e o demandante como forma de se atingir uma formação profissional de qualidade. Excetuam-se os MPs em rede, que, por se tratarem de programas para atender a áreas consideradas estratégicas para o país, têm sido induzidos pela Capes, a exemplo do Profmat (matemática), do Profletras (letras), do Profis (física), do Profhist (história), do Profartes (artes) e do Profiap (administração pública), para os quais a entidade prevê formas de participação financeira. Os demais não recebem bolsas ou incentivos do órgão federal.

O crescimento da oferta de MP é expressivo. No entanto, o grande desafio é a manutenção da qualidade na oferta e a expansão do MP para regiões do país nas quais ainda existe um número de programas profissionais muito reduzido, como Norte, Nordeste e Centro-Oeste.

As assimetrias no sistema de pós-graduação brasileiro não são exclusividade do MPe têm sido apontadas desde o PNPG 2005-2010 (Capes, 2005a). Persistem e são reforçadas no programaparaadécadaseguinte(Capes, 2010).Notadamente, asmesorregiõesmetropolitanas de São Paulo e do Rio de Janeiro apresentam indicadores destacados das demais e têm alta oferta de programas de pós-graduação. Para mudar esse quadro, torna-se oportuno levar em consideração o estabelecimento de relações com parceiros para a definição de projetos sustentáveis, cuja complexidade irá exigir a ação sinérgica de vários órgãos.

No que se refere à oferta de MP em rede, o campo da saúde coletiva vem acumulando importante experiência por meio da Rede Nordeste de Formação em Saúde da Família (Renasf), que emerge em 2009, constituída por 23 instituições de educação média e superior, instituições de pesquisa e serviços de saúde da região Nordeste. No âmbito dessa rede, nasceu 
a proposta de um curso stricto sensu para profissionais da Estratégia Saúde da Família da região - que culminou em 2010 com o credenciamento do Curso de Mestrado Profissional em Saúde da Família pela Capes. A primeira turma iniciou suas atividades em 2012 (Hortale et al., no prelo).

Aestrutura organizacional desseMP desenvolveu-se pormeio deumaamplaassociação entre as universidades Federal do Rio Grande do Norte, Estadual do Vale do Acaraú, Estadual do Ceará, Federal do Ceará, Federal do Maranhão e a Fundação Oswaldo Cruz, incorporando ainda docentes e infraestrutura de ensino e pesquisa de outras instituições associadas à Renasf. As instituições envolvidas foram convidadas a participar dos diversos encontros com o objetivo de definir as bases teórico-práticas de ensino-aprendizagem que auxiliaram na construção do curso, de modo a contribuir para o fortalecimento da produção do conhecimento das instituições associadas à rede (Hortale et al., no prelo).

Como consequência de todo esse movimento, o MP em saúde da família se concretizou atendendo às políticas deeducação permanente e de formação de profissionais dos serviços, tendo como prioridade fortalecer a formação técnico-científica do profissional da saúde inserido na atenção primária - incentivando-o ao uso do método e de evidências científicas para a tomada de decisões na gestão do cuidado e do processo de trabalho (Machado et al., no prelo).

\section{Considerações Finais}

Se a formação profissional stricto sensu demorou a ser instituída no país, a sua experiência, embora recente em termos históricos, pode ser considerada exitosa. Detal forma que se discute atualmente, no âmbito da Capes, a recomendação de mestrados acadêmicos apenas como etapa para o doutorado, tornando todos os demais mestrados profissionais. Também em debate encontra-se a criação de doutorados profissionais.

O MP caracteriza-se como a modalidade de excelência para formar profissionais que respondama questões estratégicas para a saúde coletiva tais como:avaliação de tecnologias em saúde,economiadasaúde, comunicaçãosocialesaúde, direitosanitário,epidemiologiagenética.

Goldbaum (2006) ressalta que os recursos humanos formados deverão ser atualizados, para serem capazes de acompanhar e incorporar os processos de inovação e produção de conhecimentos e formular projetos para responder aos problemas identificados na área de atuação, além de avaliar o impacto dessas intervenções aplicadas.

A conformação atual do mercado e das práticas profissionais, com maior rotatividade dos postos de trabalho e alta incorporação tecnológica, requer um profissional formado para a transferência mais rápida dos conhecimentos adquiridos na academia para a sociedade. Anecessidadedeintegraçãoensino-serviçovem sendo discutida háalgumtempo, comoforma de superar os modelos tradicionais de formação naárea da saúde, quais sejam:o"modoescolar, 
distanciado da realidade concreta, e o modo serviço, nela mergulhado"(Teixeira, 2006:41). A modalidade de formação pós-graduada profissional é uma das melhores possibilidades de resposta a esse desafio.

O envolvimento da coletividade da Abrasco com o desenvolvimento dos MPs - que setem traduzido notrabalho permanentementerealizado pelos coordenadores de programas no Fórum de Coordenadores de Pós-Graduação em Saúde Coletiva - também se manifesta mais recentemente no envolvimento direto da associação na elaboração de uma proposta de MP em saúde da família em rede nacional. Trata-se de uma proposta inovadora, formulada em sintonia com os objetivos do Programa Mais Médicos, que prevê aperfeiçoar médicos e outros profissionais para atuação no desenvolvimento de políticas públicas de saúde do país e na organização e no funcionamento do SUS. As atividades didático-pedagógicas do MP em saúde da família seriam desenvolvidas por instituições associadas em rede nacional e coordenadas pela própria Abrasco.

Nos seus 35 anos de existência a Abrasco mantém o seu compromisso com a formação de sujeitos comprometidos com os princípios e valores da Reforma Sanitária brasileira e com o aperfeiçoamento do SUS.

\section{REFERÊNCIAS}

BRASIL. Conselho Federal de Educação. Parecer n. 977 CES, de 3 dez. 1965. Define os cursos de pósgraduacão. Disponível em:<http://nucleodememoria.vrac.puc-rio.br/site/textosfinais/parecerCFE97765. pdf >. Acesso em: 10 ago. 2014.

COORDENAÇÃO DE APERFEIÇOAMENTO DE PESSOAL DE NÍVEL SUPERIOR (CAPES). Portaria n. 80, de $16 \mathrm{dez}$. 1998. Dispõe sobre o reconhecimento dos mestrados profissionais e dá outras providências.Disponívelem:<http://ebape.fgv.br/sites/ebape.fgv.br/files/portaria_capes_080.pdf>.Acesso em: 10 ago. 2014.

COORDENAÇÃO DE APERFEIÇOAMENTO DE PESSOAL DE NÍVEL SUPERIOR (CAPES). V Plano Nacional de Pós-Graduação (PNPG) 2005-2010. Brasília: Capes, 2005a.

COORDENAÇÃO DE APERFEIÇOAMENTO DE PESSOAL DE NÍVEL SUPERIOR (CAPES). Portaria n. 47, de 17 out. 1995. Determina a implantação na Capes de procedimentos apropriados à recomendação, acompanhamento e avaliação de cursos de mestrado dirigidos à formação profissional. Revista Brasileira de Pós-Graduação, 2(4): 147-148, 2005b. Disponível em: <www.foprof.org.br/documentos/portaria-no47-17-outubro-1995.pdf>. Acesso em: 14 ago. 2014.

COORDENAÇÃO DE APERFEIÇOAMENTO DE PESSOAL DE NÍVEL SUPERIOR (CAPES). Programa de flexibilização do modelo de pós-graduação senso estrito em nível de mestrado-1995. Revista Brasileira de Pós-Graduação, 2(4): 145-146, 2005c. Disponível em: <http://ojs.rbpg.capes.gov.br/index. php/rbpg/article/viewFile/86/82>. Acesso em: 14 ago. 2014.

COORDENAÇÃO DE APERFEIÇOAMENTO DE PESSOAL DE NÍVEL SUPERIOR (CAPES). Portaria normativa n. 17, de 28 dez. 2009. Dispõe sobre o mestrado profissional no âmbito da Fundação Coordenação de Aperfeiçoamento de Pessoal de Nível Superior - Capes. Disponível em: <http://portal. iphan.gov.br/baixaFcdAnexo.do?id=3922 >. Acesso em: 12 ago. 2014. 
COORDENAÇÃO DE APERFEIÇOAMENTO DE PESSOAL DE NÍVEL SUPERIOR (CAPES). Plano Nacional de Pós-Graduação - PNPG 2011-2020: coordenação de pessoal de nível superior. Brasília: Capes, 2010. 2 v.

COORDENAÇÃO DE APERFEIÇOAMENTO DE PESSOAL DE NÍVEL SUPERIOR (CAPES). Portaria n. 1, de 4 jan. 2012. Define, para efeitos da avaliação, realizada pela CAPES, a atuação nos diferentes programas e cursos de pós-graduação das diferentes categorias de docentes. Disponível em: <www.capes. gov.br/images/stories/.../Portaria-1_4-jan-2012.pdf>. Acesso em: 17 abr. 2015.

COORDENAÇÃO DE APERFEIÇOAMENTO DE PESSOAL DE NÍVEL SUPERIOR (CAPES). Relação de cursos recomendados e reconhecidos, 2014a. Disponível em: <http://conteudoweb.capes.gov.br/ conteudoweb/ProjetoRelacaoCursosServlet?acao=pesquisarAreaAvaliacao\#>. Acesso em: 16 ago. 2014.

COORDENAÇÃO DE APERFEIÇOAMENTO DE PESSOAL DE NÍVEL SUPERIOR (CAPES). Documento de área 2013: saúde coletiva, 2014b. Disponível em: <www.capes.gov.br/images/ stories/download/avaliacaotrienal/Docs_de_area/Saude_Coletiva_doc_area_e_comiss\%C3\%A3o_ att08deoutubro.pdf >. Acesso em: 14 ago. 2014.

FISCHER, T. Educação profissional: proposições sobre educação profissional em nível de pós-graduação para o PNPG 2011-2020 In: BRASIL. Ministério da Educação. Coordenação de Aperfeiçoamento de Pessoal de Nível Superior. Plano Nacional de Pós-Graduação - PNPG 2011-2020: coordenação de pessoal de nível superior. v. 2. Brasília: Capes, 2010.

FONSECA, C. M. O. A história da Abrasco: política, ensino e saúde no Brasil. In: LIMA, N. T. \& SANTANA, J. P. (Orgs.). Saúde Coletiva como Compromisso a Trajetória da Abrasco. Rio de Janeiro: Editora Fiocruz, Abrasco. 2006.

FRENK, J. et al. Health professionals for a new century: transforming education to strengthen health systems in an interdependent world. The Lancet, 376(9.756): 1.923-1.958, 2014.

GOLDBAUM, M. Mestrado profissionalizante em saúde coletiva. In: LEAL, M. C. \& FREITAS, C. M. (Orgs.).Cenários Possíveis: experiências e desafios do mestrado profissional na saúde coletiva. Rio de Janeiro: Editora Fiocruz, 2006.

GOLDBAUM, M. \& BARATA, R. B. O feito por fazer. In: LIMA, N. T. \& SANTANA, J. P. (Orgs.). Saúde Coletiva como Compromisso a Trajetória da Abrasco. Rio de janeiro: Editora Fiocruz, Abrasco, 2006.

HARTZ, Z. M. A. \& NUNES, T. C. M. Formação e capacitação dos recursos humanos no Brasil: situação atual, desafios e perspectivas da pós-graduação em saúde coletiva. In: LEAL, M. C. \& FREITAS, C. M. (Orgs.).Cenários Possíveis: experiências e desafios do mestrado profissional na saúde coletiva. Rio de Janeiro: Editora Fiocruz, 2006.

HORTALE, V. A. Mestrado profissionalizante em saúde pública. In: LEAL, M. C. \& FREITAS, C. M. (Orgs.).Cenários Possíveis: experiências e desafios do mestrado profissional na saúde coletiva. Rio de Janeiro: Editora Fiocruz, 2006.

HORTALE, V. A. et al. Mestrado profissional em saúde da família: construção teórico-metodológica e aprendizados com a experiência. Revista Trabalho, Educação e Saúde, no prelo.

MACHADO, M. F. A. S. et al. Mestrado profissional em saúde da família: o processo de construção do currículo. Revista Trabalho, Educação e Saúde, no prelo.

MESTRADO NO BRASIL. A situação e uma nova perspectiva. Infocapes, 3(3-4): 18-21, 1995. Disponível em: <www.scielo.br/pdf/rac/v1n2/v1n2a08.pdf>. Acesso em: 10 ago. 2014.

MINAYO, M. C. S. A atuação da Abrasco em relação ao ensino de pós-graduação na área de saúde coletiva. In: LIMA, N. T. \& SANTANA, J. P. (Orgs.). Saúde Coletiva como Compromisso a Trajetória da Abrasco. Rio de Janeiro: Editora Fiocruz, Abrasco, 2006. 
PAGLIOSA, F. L. \& DA ROS, M. A. O Relatório Flexner: para o bem e para o mal. Revista Brasileira de Educação Médica, 32(4): 492-499, 2008.

PAIM, J. S. \& PINTO, I. C. M. Graduação em saúde coletiva: conquistas e passos para além do sanitarismo. Tempus - Actas Saúde Coletiva, 7: 14-35, 2013.

QUELHAS, O. L. G.; FARIA FIHO, J. R. \& FRANÇA, S. L. B. O mestrado profissional no contexto do sistema de pós-graduação brasileiro. Revista Brasileira de Pós-Graduação, 2(4): 97-104, 2005.

RIBEIRO, R. J. Ainda sobre o mestrado profissional. Revista Brasileira de Pós-Graduação, 3(6): 313-315, 2006.

SANTOS, G. B.; HORTALE, V. A. \& AROUCA, R. Mestrado Profissional em Saúde Pública. Rio de Janeiro: Editora Fiocruz, 2012.

TEIXEIRA, C. Significado estratégico do mestrado profissionalizante na consolidação do campo da saúde coletiva. In: LEAL, M. C. \& FREITAS, C. M. (Orgs.). Cenários Possíveis: experiências e desafios do mestrado profissional na saúde coletiva. Rio de Janeiro: Editora Fiocruz, 2006. 